\title{
On the Light Massive Flavor Dependence of the Top Quark Mass
}

\author{
André H. Hoang ${ }^{1,2, \star}$, Christopher Lepenik ${ }^{1, \star \star}$, and Moritz Preisser ${ }^{1, \star \star \star}$ \\ ${ }^{1}$ University of Vienna, Faculty of Physics Boltzmanngasse 5, A-1090 Wien, Austria \\ ${ }^{2}$ Erwin Schrödinger International Institute for Mathematical Physics, University of Vienna, Boltzmanngasse \\ 9, A-1090 Wien, Austria
}

\begin{abstract}
We provide a systematic renormalization group formalism for the mass effects in the relation of the pole mass and short-distance masses such as the $\overline{\mathrm{MS}}$ mass of a heavy quark $Q$, coming from virtual loop insertions of massive quarks lighter than $Q$ with the main focus on the top quark. The formalism reflects the constraints from heavy quark symmetry and entails a combined matching and evolution procedure that allows to disentangle and successively integrate out the corrections coming from the lighter massive quarks and the momentum regions between them and also to precisely control the large order asymptotic behavior. With the formalism we systematically sum logarithms of ratios of the lighter quark masses and heavy quark mass, predict the $O\left(\alpha_{s}^{4}\right)$ virtual quark mass corrections in the pole- $\overline{\mathrm{MS}}$ mass relation and calculate the pole mass differences for the top, bottom and charm quarks with a precision of around $20 \mathrm{MeV}$.
\end{abstract}

\section{Introduction}

The masses of the heavy quarks, especially of the top, are among the most important parameters in the standard model, having strong impact on e.g. precise consistency tests of the standard model and the estimation of the electroweak vacuum stability. However, it must be kept in mind that the mass of a heavy quark $Q$, due to confinement, is not a physical observable but should be viewed as a formal theory parameter which depends on the renormalization scheme. This concept is incorporated most cleanly in the so-called $\overline{\mathrm{MS}}$ mass $\bar{m}_{Q}(\mu)$, which is defined analogous to the $\overline{\mathrm{MS}}$-renormalized strong coupling constant $\alpha_{s}(\mu)$. Like the strong coupling, the $\overline{\mathrm{MS}}$ mass $\bar{m}_{Q}(\mu)$ depends on a renormalization scale $\mu$. This scale can be interpreted as the scale above which short-distance information is contained in the mass and should be chosen around or higher than the mass scale. The $\overline{\mathrm{MS}}$ mass has no kinematic meaning, but can be measured very precisely from experimental data. On the other hand, the socalled pole mass $m_{Q}^{\text {pole }}$ is defined as the position of the single particle pole of correlation functions containing the heavy quark $Q$ as an external on-shell state, which is identical to the statement that in the pole mass scheme all on-shell self-energy corrections are absorbed into the mass parameter, including contributions from energy regions below $1 \mathrm{GeV} \gtrsim \Lambda_{\mathrm{QCD}}$. Since the $\overline{\mathrm{MS}}$ mass does not have such contributions, it is called a "short-distance mass". The pole mass $m_{Q}^{\text {pole }}$ can be interpreted as the kinematic mass of an isolated heavy quark $Q$ in the context of perturbation theory. It is obvious

\footnotetext{
^e-mail: andre.hoang@univie.ac.at

$\star \star$ Speaker, e-mail: christopher.lepenik@univie.ac.at

$\star \star \star$ e-mail: moritz.preisser@univie.ac.at
} 
that the definition of the pole mass implies some conceptual and practical problems, since, due to confinement, external on-shell quarks do not exist and therefore they do not arise as single particle poles in physical correlation functions. Nevertheless it is unavoidable that the pole mass scheme appears in one way or another in higher order QCD calculations involving external massive quarks. For perturbative calculations involving the production of top quarks at hadron colliders, the pole mass scheme is the main top quark mass scheme used in the literature.

We now investigate the perturbative behavior of the pole mass for the top, bottom and charm quarks. For this purpose it is convenient to consider the perturbation series relating the pole and $\overline{\mathrm{MS}}$ mass. In Refs. [1-8] this relation was computed to 4-loop accuracy, within the approximation that all quarks lighter then $Q$ are massless. Using their results and the top, bottom and charm quark $\overline{\mathrm{MS}}$ mass values $\bar{m}_{t} \equiv \bar{m}_{t}\left(\bar{m}_{t}\right)=163 \mathrm{GeV}, \bar{m}_{b} \equiv \bar{m}_{b}\left(\bar{m}_{b}\right)=4.2 \mathrm{GeV}$ and $\bar{m}_{c} \equiv \bar{m}_{c}\left(\bar{m}_{c}\right)=1.3 \mathrm{GeV}$ we obtain

$$
\begin{aligned}
& m_{t}^{\text {pole }}=163+7.5040+1.6005+0.4941+(0.1944 \pm 0.0004) \mathrm{GeV}, \\
& m_{b}^{\text {pole }}=4.2+0.3998+0.1986+0.1443+(0.1349 \pm 0.0002) \mathrm{GeV}, \\
& m_{c}^{\text {pole }}=1.3+0.2108+0.1984+0.2725+(0.4843 \pm 0.0005) \mathrm{GeV},
\end{aligned}
$$

where the terms show the series in powers of the strong coupling $\alpha_{s}\left(\bar{m}_{Q}\right)$ in the scheme that includes $Q$ as a dynamical flavor. The fourth order coefficient displays the numerical uncertainties from [8], which are, however, much smaller than other types of uncertainties considered here.

The pole mass scheme is infrared-save and gauge-invariant [1, 9], but suffers from large perturbative corrections, as can be seen explicitly already at low orders from the series above. The bad perturbative behavior is related to the absorption of linearly infrared sensitive on-shell self-energy contributions into the pole mass. This infrared sensitivity of the on-shell diagrams grows with loop order. In the large- $\beta_{0}$ limit, the asymptotic large order behavior of the pole- $\overline{\mathrm{MS}}$ relation has the form

$$
m_{Q}^{\text {pole }}-\bar{m}_{Q}\left(\bar{m}_{Q}\right) \sim \mu \sum_{n=0}^{\infty} \frac{16}{3}\left(2 \beta_{0}^{\left(n_{\ell}\right)}\right)^{n} n !\left(\frac{\alpha_{s}^{\left(n_{\ell}\right)}(\mu)}{4 \pi}\right)^{n+1},
$$

where $\beta_{0}$ is defined via the $\mathrm{QCD} \beta$-function describing the running of the strong coupling

$$
\frac{\mathrm{d} \alpha_{s}^{\left(n_{\ell}\right)}(\mu)}{\mathrm{d} \log \mu}=\beta^{\left(n_{\ell}\right)}\left(\alpha_{s}(\mu)\right)=-2 \alpha_{s}^{\left(n_{\ell}\right)}(\mu) \sum_{n=0}^{\infty} \beta_{n}^{\left(n_{\ell}\right)}\left(\frac{\alpha_{s}^{\left(n_{\ell}\right)}(\mu)}{4 \pi}\right)^{n+1} .
$$

The variable $n_{\ell}$ denotes the number of quarks lighter then $\Lambda_{\mathrm{QCD}}$, which are taken to be massless throughout this work. The pattern of factorial divergence of the perturbation series and the linear dependence on the renormalization scale $\mu$ of the strong coupling are called the $O\left(\Lambda_{\mathrm{QCD}}\right)$ renormalon of the pole mass $[10,11]$. It is an important observation that, up to power corrections of order $\Lambda_{\mathrm{QCD}}^{2} / m_{Q}$, the asymptotic large order behavior is independent of the heavy quark mass $m_{Q}$. This follows from Heavy Quark Symmetry (HQS) [12]: low momentum sensitivity grows with loop order and the high order terms are therefore governed almost exclusively by long-distance physics. Consequently, in this regime the heavy quarks are essentially static color sources whose precise mass values are irrelevant. On the other hand, it should be stressed that the renormalon is solely an artifact of the pole mass definition and not related to any physical effects. Another crucial point is that the high order asymptotics depends only on the number of massless quark flavors $n_{\ell}$. This can again be understood from the HQS argument above. Due to the linear dependence of Eq. (4) to the renormalization scale $\mu$ the precise value which is chosen in practice is of high importance. E.g., to ensure the cancellation of an $O\left(\Lambda_{\mathrm{QCD}}\right)$ renormalon between two series, the same $\mu$-value must be chosen. We note that in the relation between the pole and other short-distance masses the same pattern can be seen: at asymptotic 
high orders one observes factorial growth and the series becomes linear in the renormalization scale of the stong coupling.

It is important to note that the renormalon behavior of the pole mass is not just an issue of pedagogical interest, but one that is relevant phenomenologically [13]. This is because for $\mu=\bar{m}_{Q}$ the known coefficients of the series in Eqs. (1)-(3) agree remarkably well with the corresponding large order asymptotic behavior already beyond the terms of $O\left(\alpha_{s}\right)$ (so that the terms of the series are known quite precisely to all orders) and because even for orders where the QCD corrections still decrease with order they can be very large numerically and make phenomenological applications difficult.

Although the perturbative series relating the pole and $\overline{\mathrm{MS}}$ mass is diverging, the series is asymptotic and therefore not meaningless. It is known from the theory of asymptotic series that the best approximation of the LHS of series like in Eq. (4) is usually obtained when truncating the asymptotic series at the smallest correction term at order $n_{\min }$. There is a region in the orders $n$ around $n_{\min }$ of width $\Delta n$ in which all series terms have a size close to the minimal term. At orders above $n_{\min }+\Delta n / 2$ the series diverges quickly and the series terms from these orders are useless even if they are known through an elaborate loop calculation. The uncertainty with which the pole mass can be determined in principle given the full information about the perturbative series is called the pole mass ambiguity. It is universal, independent of the choice of the renormalization scale $\mu$ and exists in equivalent size in any context without the possibility to be circumvented. Using the method of Borel summation the pole mass ambiguity can be estimated to be of order $\Lambda_{\mathrm{QCD}}^{\left(n_{\ell}\right)}$, where the superscript $\left(n_{\ell}\right)$ stands for the dependence of the hadronization scale on the number of massless quark flavors.

For more details on the numerical values and derivations we refer to Ref. [14].

\section{Light Massive Flavor Dependence}

Due to the hierarchy in quark masses, in many applications of top quark physics the bottom and charm quarks may be taken as massless. However, when interested in the high order behavior of the pole mass, due to the increasing sensitivity to low scale physics, the effects of lighter massive quarks $q$ with $m_{q}>\Lambda_{\mathrm{QCD}}$ become relevant and actually have some impact. The mass of a virtual quark flavor in the on-shell self-energy diagram acts as an effective infrared cut-off at the mass scale and therefore this quark flavor is removed from the high order asymptotic behavior [15]. The removal of heavy lighter flavors from the high order asymptotics together with the $n_{\ell}$-dependence of the QCD $\beta$-function lead to an increased infrared sensitivity of the heavy quark pole mass and a stronger divergence pattern of the series, as can be seen from Eq. (4). It is obvious that, since the masses of the lighter massive flavors alter the pattern of divergence of the renormalon series, their induced corrections are themselves plagued by a renormalon.

To study these corrections it is again convenient to consider the perturbative relation between the pole and $\overline{\mathrm{MS}}$ mass. Including the bottom and charm quark as massive flavors, the pole- $\overline{\mathrm{MS}}$ mass relation for the top quark can be written in the form [14]

$$
m_{t}^{\text {pole }}-\bar{m}_{t}=\bar{m}_{t} \sum_{n=1}^{\infty} a_{n}\left(n_{t}+1\right)\left(\frac{\alpha_{s}^{\left(n_{t}+1\right)}\left(\bar{m}_{t}\right)}{4 \pi}\right)^{n}+\bar{m}_{t}\left[\bar{\delta}_{t}^{(t, b, c)}\left(1, r_{b t}, r_{c t}\right)+\bar{\delta}_{t}^{(b, c)}\left(r_{b t}, r_{c t}\right)+\bar{\delta}_{t}^{(c)}\left(r_{c t}\right)\right],
$$

where $\bar{m}_{t} \equiv \bar{m}_{t}\left(\bar{m}_{t}\right), n_{t}=5$ denotes the number of quark flavors lighter than the top, $a_{n}\left(n_{t}+1\right)$ are the perturbative coefficients describing the QCD corrections from gluons and $n_{t}+1$ massless virtual quarks, and $\alpha_{s}^{\left(n_{t}+1\right)}$ is the strong coupling that evolves with $n_{t}+1$ active dynamical flavors, see Eq. (5). Results for the corrections from one lighter massive flavor up to $O\left(\alpha_{s}^{3}\right)$ were determined [2, 16]. The terms $\bar{\delta}_{t}$ contain the mass corrections coming from the top quark on-shell self-energy diagrams with insertions of virtual massive quark loops and can be written in a perturbative expansion in $\alpha_{s}^{\left(n_{t}+1\right)}$. The 
superscripts of the form $\left(q, q^{\prime}, \ldots\right)$ indicate that each diagram contains at least one insertion of the massive quark $q$ and in addition all possible insertions of the (lighter) massive quarks $q^{\prime}, \ldots$ as well as of massless quark and gluonic loops. $b$ and $c$ relate to the bottom and charm quark respectively. From each diagram the corresponding diagram with all the quark loops in the massless limit is subtracted in the scheme compatible with the flavor number scheme for the strong coupling $\alpha_{s}$. The fraction $r_{q q^{\prime}} \equiv \bar{m}_{q} / \bar{m}_{q^{\prime}}$ stands for the ratio of $\overline{\mathrm{MS}}$ masses for quarks $q$ and $q^{\prime}$. By construction, the sum of all virtual quark mass corrections contained in the functions $\bar{\delta}_{t}$ are RG-invariant: they originate solely from the quark self-energy diagrams arising in the pole mass renormalization and, since the $\bar{\delta}_{t}$ denote the difference between massive and massless diagrams, the UV-divergences are canceled.

The aim now is to study the contributions of the light massive flavors in a coherent and systematic manner, which is done in a renormalization group framework. This framework allows to disentangle the contributing momentum modes and resum the logarithms of quark mass ratios which arise in the multi-scale problem. Consequently, it is possible to study the large order behavior and general structure of the mass corrections and the overall large order behavior of the pole mass, and use this knowledge in several applications.

\section{Integrating Out Hard Modes from the Top Quark Pole Mass}

\subsection{MSR Mass}

To set up the renormalization group framework and disentangle the different momentum regions below the top mass scale, we use the MSR mass $m_{Q}^{\mathrm{MSR}}(R)$ which was introduced in Ref. $[17,18]$, however, without the inclusion of massive lighter quarks. In the presence of massive bottom and charm quarks the top quark MSR mass is defined through [14]

$$
m_{t}^{\mathrm{pole}}-m_{t}^{\mathrm{MSR}}(R)=R \sum_{n=1}^{\infty} a_{n}\left(n_{t}\right)\left(\frac{\alpha_{s}^{\left(n_{t}\right)}(R)}{4 \pi}\right)^{n}+\bar{m}_{t}\left[\delta_{t}^{(b, c)}\left(r_{b t}, r_{c t}\right)+\delta_{t}^{(c)}\left(r_{c t}\right)\right]
$$

where the coefficients $a_{n}$ are the same as in the pole- $\overline{\mathrm{MS}}$ relation Eq. (6) and $R$ is a momentum scale which is in principle arbitrary, but should be sufficiently larger than $\Lambda_{\mathrm{QCD}}$ to stay away from the Landau pole. The terms $\delta_{t}$ are derived from the respective $\bar{\delta}_{t}$ of Eq. (6) by replacing $\alpha_{s}^{\left(n_{t}+1\right)} \rightarrow \alpha_{s}^{\left(n_{t}\right)}$ in the perturbative expansions. The introduction of the MSR mass is necessary since the $\overline{\mathrm{MS}}$ mass is not adequate to describe scales far below the heavy quark mass scale. The MSR mass fixes the two main problems in this context: first, the heavy quark is removed as a dynamical degree of freedom from the series (i.e. integrated out). Second, the MSR mass incorporates linear scale dependence by replacing $\bar{m}_{t}$ multiplying the coefficients $a_{n}$ on the RHS of Eq. (6) with the arbitrary momentum scale $R$. Linear scale dependence is crucial in the low momentum region to describe the linear low momentum sensitivity of the pole mass. Indeed linear momentum dependence appears also in the pole- $\overline{\mathrm{MS}}$ mass relation, but only at asymptotically high orders (see Eq. (4)). It should be also mentioned that the replacement of $\bar{m}_{t}$ and the removal of the heavy flavor in the definition of the MSR mass do not change the asymptotic high order behavior of the series, because the latter is independent of the heavy quark mass and the number of massive flavors. As argued at the end of Sec. 2, the mass corrections are RG-invariant and it is therefore natural to leave the mass correction term independent of $R$. So the MSR mass is defined in close analogy to the $\mu$-dependent $\overline{\mathrm{MS}}$ strong coupling whose renormalization group evolution only depends on the number of active dynamical quarks (which is typically the number of quarks lighter than $\mu$ ) and where mass effects are implemented by threshold corrections when $\mu$ crosses a flavor threshold. 

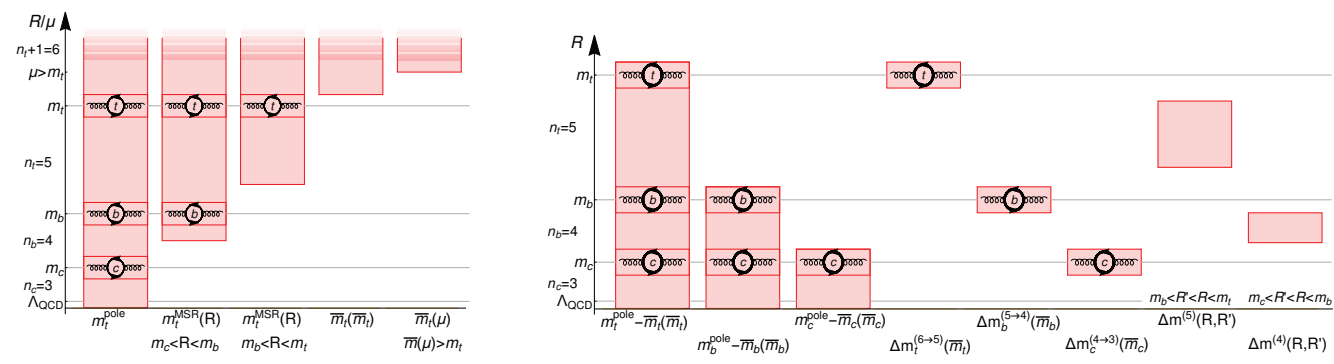

Figure 1. Left: Graphical illustration of the physical contributions contained in the pole, MSR and $\overline{\mathrm{MS}}$ mass schemes coming from the different momentum scales for the case of the top quark. The quark loops stand for the contributions of the virtual massive quark loops contained in the masses. Right: Graphical illustration for pole- $\overline{\mathrm{MS}}$ mass differences, the MSR- $\overline{\mathrm{MS}}$ mass matching corrections and MSR mass differences for different $R$ scales. They constitute the major contributions in the RG analysis of the heavy quark pole masses.

The resulting renormalization group equation in $R$ is linear in $R$, as required by low scale physics, and is called the R-evolution equation. For an arbitrary heavy quark flavor $Q$, it takes the form

$$
R \frac{\mathrm{d}}{\mathrm{d} R} m_{Q}^{\mathrm{MSR}}(R)=-R \gamma^{R,\left(n_{Q}\right)}\left(\alpha_{s}^{\left(n_{Q}\right)}(R)\right)=-R \sum_{n=0}^{\infty} \gamma_{n}^{R,\left(n_{Q}\right)}\left(\frac{\alpha_{s}^{\left(n_{Q}\right)}(R)}{4 \pi}\right)^{n+1},
$$

where $n_{Q}$ denotes the number of quark flavors lighter than $Q$ and the coefficients $\gamma_{n}^{R,\left(n_{Q}\right)}$ are known up to four loops and given in Refs. [17, 18]. It is easy to see that Eq. (8) is renormalon-free since the renormalon ambiguity of the series proportional to $R$ is independent of $R$ and therefore canceled when differentiated.

As mentioned below Eq. (5), it is crucial to choose the same renormalization scale when subtracting two series which contain an $O\left(\Lambda_{\mathrm{QCD}}\right)$ renormalon. This is e.g. the case for the difference of the MSR mass at two different $R$-scales in fixed-order perturbation theory using Eq. (7), which for widely separated scales leads inevitably to large logarithms. To avoid this problem one can use the solution of the R-evolution equation Eq. (8)

$$
\Delta m^{\left(n_{Q}\right)}\left(R, R^{\prime}\right)=m_{Q}^{\mathrm{MSR}}\left(R^{\prime}\right)-m_{Q}^{\mathrm{MSR}}(R)=\sum_{n=0}^{\infty} \gamma_{n}^{R,\left(n_{Q}\right)} \int_{R^{\prime}}^{R} \mathrm{~d} R\left(\frac{\alpha_{s}^{\left(n_{Q}\right)}(R)}{4 \pi}\right)^{n+1},
$$

to resum these logarithms in a renormalon free way.

One can interpret the MSR mass as the pole mass minus all self-energy contributions coming from scales below $R$ and all virtual quark mass corrections from quarks lighter than the heavy quark, see the left plot of Fig. 1. This also illustrates that the MSR mass is a short-distance mass. $\Delta m^{\left(n_{Q}\right)}\left(R, R^{\prime}\right)$ represents the self-energy contributions to the mass in the presence of $n_{Q}$ active dynamical flavors coming from the scales between $R^{\prime}$ and $R$. This is illustrated in the right plot of Fig. 1.

\subsection{MSR-MS Mass Matching}

The matching relation between the top MSR and $\overline{\mathrm{MS}}$ mass at the common scale $\mu=R=\bar{m}_{t}$ can be obtained by eliminating the pole mass from Eqs. (6) and (7). The resulting matching coefficient then 
accounts for the virtual top quark contributions and can be written in the form

$$
m_{t}^{\mathrm{MSR}}\left(\bar{m}_{t}\right)-\bar{m}_{t}=\Delta m_{t}^{\left(n_{t}+1 \rightarrow n_{t}\right)}\left(\bar{m}_{t}\right)+\delta m_{t, b, c}^{\left(n_{t}+1 \rightarrow n_{t}\right)}\left(\bar{m}_{t}\right) .
$$

The term $\Delta m_{t}^{\left(n_{t}+1 \rightarrow n_{t}\right)}\left(\bar{m}_{t}\right)$ contains the virtual top quark loop contributions in the approximation that all $n_{t}$ quarks lighter than the top are massless and can be expressed in a power series in the strong coupling in the $\left(n_{t}+1\right)$ flavor scheme. The series only contains the hard corrections coming from the virtual heavy top and therefore does not have any $O\left(\Lambda_{\mathrm{QCD}}\right)$ ambiguity, see the right plot of Fig. 1 for illustration. $\Delta m_{t}^{\left(n_{t}+1 \rightarrow n_{t}\right)}\left(\bar{m}_{t}\right)$ can therefore be computed to high precision using the $a_{n}$ coefficients which are known up to $O\left(\alpha_{s}^{4}\right)[1-8]$.

For the top quark the matching corrections amount to approximately $30 \mathrm{MeV}$ and the analogous corrections for the bottom and charm amount to approximately $5 \mathrm{MeV}$, with an uncertainty at the level of 1 to $2 \mathrm{MeV}$.

The term $\delta m_{t, b, c}^{\left(n_{t}+1 \rightarrow n_{t}\right)}\left(\bar{m}_{t}\right)$ represents the virtual top quark contributions arising from the finite masses of the lighter massive bottom and charm quarks. Since at $O\left(\alpha_{s}^{2}\right)$ only the loop of a top quark can be inserted, the series for $\delta m_{t, b, c}^{\left(n_{t}+1 \rightarrow n_{t}\right)}\left(\bar{m}_{t}\right)$ starts at $O\left(\alpha_{s}^{3}\right)$, where only self-energy diagrams with one insertion of a top quark loop and one insertion of a loop of one of the lighter massive quarks can contribute. The expansions of $\delta m_{t, b, c}^{\left(n_{t}+1 \rightarrow n_{t}\right)}\left(\bar{m}_{t}\right)$ in the mass ratios $r_{b t}$ and $r_{c t}$ start quadratically in these ratios indicating that the corrections are governed by the scale $\bar{m}_{t}$ just like the matching term $\Delta m_{t}^{\left(n_{t}+1 \rightarrow n_{t}\right)}\left(\bar{m}_{t}\right)$ and do not have any linear sensitivity to small momenta and the lighter quark masses, in particular. This feature is realized at any order of perturbation theory. Because the finite mass corrections $\delta m_{t, b, c}^{n_{t}+1 \rightarrow n_{t}}\left(\bar{m}_{t}\right)$ start at $O\left(\alpha_{s}^{3}\right)$ and are quadratic in the mass ratios they are extremely small and never exceed $0.01 \mathrm{MeV}$. This is also true for the finite mass corrections to the bottom quark due to the massive charm quark and we can expect that this is also exhibited at higher orders. Hence, this kind of correction can be neglected for all practical purposes and will not be considered and discussed any further here.

\subsection{Top-Bottom and Bottom-Charm Mass Matching}

Comparing the pole-MSR mass relation for the top quark of Eq. (7) to the pole- $\overline{\mathrm{MS}}$ mass relation for the next lighter massive quark, i.e. the bottom

$$
m_{b}^{\text {pole }}-\bar{m}_{b}=\bar{m}_{b} \sum_{n=1}^{\infty} a_{n}\left(n_{b}+1\right)\left(\frac{\alpha_{s}^{\left(n_{b}+1\right)}\left(\bar{m}_{b}\right)}{4 \pi}\right)^{n}+\bar{m}_{b}\left[\bar{\delta}_{t}^{(b, c)}\left(1, r_{c b}\right)+\bar{\delta}_{t}^{(c)}\left(r_{c b}\right)\right],
$$

with $n_{b}=n_{t}-1=4$, one immediately notices that for $R=\bar{m}_{b}$ the corrections are identical in the approximation that in the virtual quark loops all $n_{t}$ lighter quarks (including the bottom quark) are treated as massless. This identity is a consequence of heavy quark symmetry which states that the low-energy QCD corrections to the heavy quark masses coming from massless partons are flavorindependent. Obviously, this is also valid when comparing the bottom to the charm quark.

The resulting matching relation reads

$$
\left[m_{t}^{\text {pole }}-m_{t}^{\mathrm{MSR}}\left(\bar{m}_{b}\right)\right]-\left[m_{b}^{\text {pole }}-\bar{m}_{b}\right]=\delta m_{b, c}^{(t \rightarrow b)}\left(\bar{m}_{b}, \bar{m}_{c}\right)
$$

where $\delta m_{b, c}^{(t \rightarrow b)}\left(\bar{m}_{b}, \bar{m}_{c}\right)$ encodes the heavy quark symmetry breaking corrections coming from the finite virtual charm and bottom quark masses. Their form can be extracted directly from Eqs. (6) and (7) and written as $\left(r_{q q^{\prime}}=\bar{m}_{q} / \bar{m}_{q^{\prime}}\right)$

$$
\delta m_{b, c}^{(t \rightarrow b)}\left(\bar{m}_{b}, \bar{m}_{c}\right)=\bar{m}_{t}\left[\delta_{t}^{(b, c)}\left(r_{b t}, r_{c t}\right)+\delta_{t}^{(c)}\left(r_{c t}\right)\right]-\bar{m}_{b}\left[\bar{\delta}_{b}^{(b, c)}\left(1, r_{c b}\right)+\bar{\delta}_{b}^{(c)}\left(r_{c b}\right)\right]
$$





Figure 2. Left: Top-MSR bottom-MS mass matching correction $\delta m_{b, c}^{(t \rightarrow b)}\left(\bar{m}_{b}, \bar{m}_{c}\right)$ at $O\left(\alpha_{s}^{2}\right)$ (red dashed curve) and $O\left(\alpha_{s}^{3}\right)$ (red solid curve) over the renormalization scale $\mu$. The virtual bottom and charm mass effects to the top quark self-energy of the first term in Eq. (13) (green curves) and the virtual bottom and charm mass effects to the bottom quark self-energy of the second term of Eq. (13) (blue curves) at $O\left(\alpha_{s}^{2}\right)$ (dashed) and $O\left(\alpha_{s}^{3}\right)$ (solid). For the masses of the top, bottom and charm quarks the values $\left(\bar{m}_{t}, \bar{m}_{b}, \bar{m}_{c}\right)=(163,4.2,1.3) \mathrm{GeV}$ are used. Right: The bottom-MSR charm-MS mass matching correction $\delta m_{c}^{(b \rightarrow c)}\left(\bar{m}_{c}\right)$ at $O\left(\alpha_{s}^{2}\right)$ (red dashed curve) and $O\left(\alpha_{s}^{3}\right)$ (red solid curve) over the renormalization scale $\mu$. The virtual charm mass effects to the bottom quark self-energy (green curves) and the virtual charm mass effects to the charm quark self-energy (blue curves) are shown at $O\left(\alpha_{s}^{2}\right)$ (dashed) and $O\left(\alpha_{s}^{3}\right)$ (solid).

where the first term on the RHS (multiplied by $\bar{m}_{t}$ ) represents the virtual bottom and charm mass effects in the top quark self-energy and the second term (multiplied by $\bar{m}_{b}$ ) represents the virtual bottom and charm mass effects in the bottom quark self-energy.

It is important that the quark mass corrections in (13) are expressed coherently in powers of $\alpha_{s}$ at the common scale $\mu$ because the individual $\delta_{n}$ terms carry infrared sensitive contributions and therefore each contain an $O\left(\Lambda_{\mathrm{QCD}}\right)$ renormalon ambiguity. In Eq. (13) these renormalon ambiguities cancel.

In the left plot of Fig. 2 the top-MSR bottom- $\overline{\mathrm{MS}}$ mass matching correction $\delta m_{b, c}^{(t \rightarrow b)}\left(\bar{m}_{b}, \bar{m}_{c}\right)$ of Eq. (12) is displayed as a function of the renormalization scale $\mu$ at $O\left(\alpha_{s}^{2}\right)$ (red dashed line) and $O\left(\alpha_{s}^{3}\right)$ (red solid line) for $\left(\bar{m}_{t}, \bar{m}_{b}, \bar{m}_{c}\right)=(163,4.2,1.3) \mathrm{GeV}$. The matching correction at $O\left(\alpha_{s}^{3}\right)$ amounts to $6 \mathrm{MeV}$ and has a scale variation of only $1 \mathrm{MeV}$ for $\bar{m}_{b} \leq \mu \leq \bar{m}_{t}$. Compared to the $O\left(\alpha_{s}^{2}\right)$ result we see a strong reduction of the scale dependence at $O\left(\alpha_{s}^{3}\right)$. An analog matching contribution $\delta m_{c}^{(b \rightarrow c)}\left(\bar{m}_{c}\right)$ can be defined, describing the heavy quark symmetry breaking corrections for the bottom quark coming from the finite charm quark mass. Again, we observe excellent convergence and strongly reduced scale dependence, see the right plot in Fig. 2.

Through successive R-evolution with the appropriate flavor number (see Eq. (9)) and matching at the mass thresholds of the bottom and charm quark (see Eqs. (10) and (12) and their generalizations to the appropriate flavors) we can now decouple the different momentum regions in the pole- $\overline{\mathrm{MS}}$ relation of Eq. (6). The resulting formula for the top quark pole mass reads

$$
\begin{aligned}
m_{t}^{\text {pole }}=\bar{m}_{t} & +\Delta m_{t}^{(6 \rightarrow 5)}\left(\bar{m}_{t}\right)+\Delta m^{(5)}\left(\bar{m}_{t}, \bar{m}_{b}\right)+\delta m_{b, c}^{(t \rightarrow b)}\left(\bar{m}_{b}, \bar{m}_{c}\right) \\
& +\Delta m_{b}^{(5 \rightarrow 4)}\left(\bar{m}_{b}\right)+\Delta m^{(4)}\left(\bar{m}_{b}, \bar{m}_{c}\right)+\delta m_{c}^{(b \rightarrow c)}\left(\bar{m}_{c}\right) \\
& +\Delta m_{c}^{(4 \rightarrow 3)}\left(\bar{m}_{c}\right)+\Delta m^{(3)}\left(\bar{m}_{c}, R\right)+R \sum_{n=1}^{\infty} a_{n}\left(n_{\ell}=3,0\right)\left(\frac{\alpha_{s}^{(3)}(R)}{4 \pi}\right)^{n},
\end{aligned}
$$


where all $\operatorname{logarithms} \log \left(\bar{m}_{b} / \bar{m}_{t}\right)$ and $\log \left(\bar{m}_{b} / \bar{m}_{c}\right)$ are systematically resummed. The sum of all terms in the first two lines is just $m_{t}^{\text {pole }}-m_{c}^{\text {pole }}+\bar{m}_{c}$, and the first term in the third line is the charm $\overline{\mathrm{MS}}$-MSR matching contribution. All quantities except for the last term are free from an $O\left(\Lambda_{\mathrm{QCD}}\right)$ renormalon ambiguity and can be evaluated to high precision with uncertainties of the order of $20 \mathrm{MeV}$ for the evolution contributions and $1 \mathrm{MeV}$ for the matching contributions using the available 4-loop expressions for $a_{n}$ [1-8] and 3-loop expressions for the mass corrections $[2,16]$. The ambiguity of the top quark pole mass due to the $O\left(\Lambda_{\mathrm{QCD}}\right)$ renormalon is contained solely in the $R$-dependent series which is just equal to $m_{c}^{\text {pole }}-m_{c}^{\mathrm{MSR}}(R)$. This relation specifies the charm quark pole mass ambiguity, and it fully encodes the top and bottom quark pole mass ambiguities due to heavy quark symmetry. It should be noted that among all the terms shown in Eq. (14) the contributions from the MSR mass differences $\Delta m^{(5)}\left(\bar{m}_{t}, \bar{m}_{b}\right), \Delta m^{(4)}\left(\bar{m}_{b}, \bar{m}_{c}\right)$ and $\Delta m^{(3)}\left(\bar{m}_{c}, R\right)$, determined with R-evolution, and the series proportional to $R$, which contains the $O\left(\Lambda_{\mathrm{QCD}}\right)$ renormalon, constitute the numerically most important terms. Their sum is of the order of $10 \mathrm{GeV}$, exceeding the contributions from the matching corrections by far, which amount to only $50 \mathrm{MeV}$.

\section{Some Applications}

\subsection{Light Virtual Quark Mass Corrections at $O\left(\alpha_{s}^{4}\right)$}

The excellent perturbative convergence of the top-MSR bottom- $\overline{\mathrm{MS}}$ mass matching correction $\delta m_{b, c}^{(t \rightarrow b)}\left(\bar{m}_{b}, \bar{m}_{c}\right)$ discussed in the previous section illustrates that it is a short-distance quantity and free of an $O\left(\Lambda_{\mathrm{QCD}}\right)$ renormalon ambiguity. This is also expected theoretically due to heavy quark symmetry. However, the facts that the overall size of the matching corrections only amounts to a few $\mathrm{MeV}$, and that the $O\left(\alpha_{s}^{3}\right)$ corrections are only around $1 \mathrm{MeV}$ as seen in Fig. 2 allows us to draw interesting conceptual implications for the large order asymptotic behavior of the virtual quark mass corrections in the mass relations of Eqs. (6) and (7). As a consequence we can predict the yet uncalculated virtual quark mass corrections at $O\left(\alpha_{s}^{4}\right)$ to within a few percent without an additional loop calculation.

Let's consider the matching correction $\delta m_{q}^{(Q \rightarrow q)}\left(\bar{m}_{q}\right)$ between the MSR mass of heavy quark $Q$ and the $\overline{\mathrm{MS}}$ mass of the next lighter massive quark $q$ assuming the massless approximation for all quarks lighter than quark $q$, i.e. $n_{Q}=n_{q}+1=n_{\ell}+1$ and $n_{\ell}=n_{q}$ being the number of massless quarks. This situation applies to the matching relation for the top-MSR and the bottom $\overline{\mathrm{MS}}$ masses for a massless charm quark or to the matching relation between the bottom-MSR and the charm- $\overline{\mathrm{MS}}$ masses.

In the left plot of Fig. 2 we have displayed separately the virtual bottom and charm mass effects to the top quark self-energy (green curves) and the virtual bottom and charm mass effects to the bottom quark self-energy (blue curves), i.e. the first and second term of the RHS of Eq. (13), at $O\left(\alpha_{s}^{2}\right)$ (dashed) and $O\left(\alpha_{s}^{3}\right)$ (solid). We see that both types of contributions each are quite large and furthermore do not at all converge. The $O\left(\alpha_{s}^{3}\right)$ corrections are even bigger than the $O\left(\alpha_{s}^{2}\right)$ corrections, which indicates that the corresponding asymptotic large order behavior already dominates the $O\left(\alpha_{s}^{2}\right)$ and $O\left(\alpha_{s}^{3}\right)$ corrections.

The origin of this behavior has been already mentioned and is understood: the mass of the virtual quark $q$ acts as an infrared cutoff and therefore modifies the infrared sensitivity of the self-energy diagrams (of quark $Q$ and of quark $q$ ) with respect to the case where the virtual loops of quark $q$ are evaluated in the massless approximation. As a consequence these corrections individually carry an $O\left(\Lambda_{\mathrm{QCD}}\right)$ renormalon ambiguity. Moreover, at large orders in perturbation theory the sensitivity of the self-energy diagrams to infrared momenta increases due to high powers of logarithms from gluonic and massless quark loops. As a consequence, at large orders, the finite mass effects of the virtual loops of quark $q$ in the self-energy diagrams of quark $Q$ and the self-energy diagrams of quark $q$ 

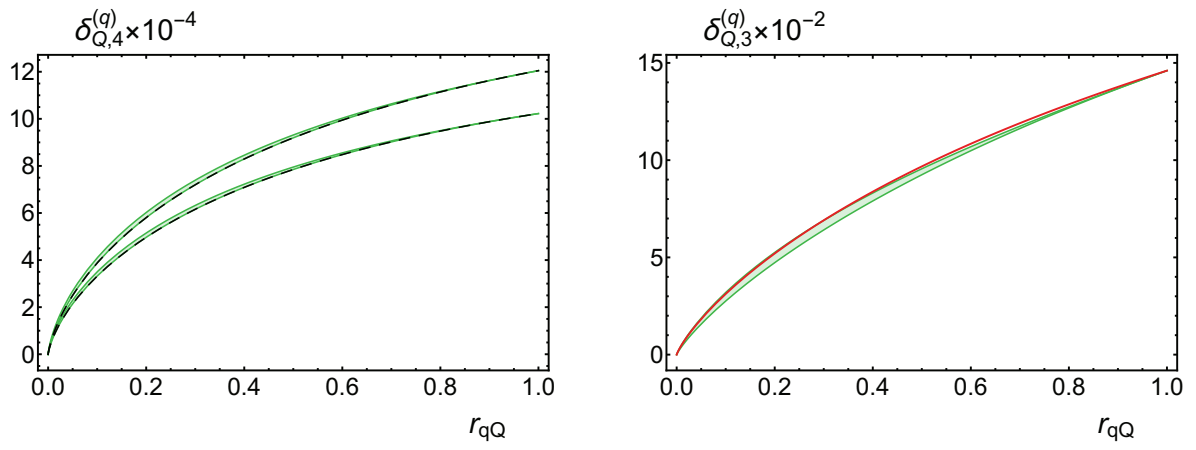

Figure 3. Left: Prediction for the $O\left(\alpha_{s}^{4}\right)$ virtual quark mass correction $\delta_{Q, 4}^{(q)}\left(r_{q Q}\right)$ for $\bar{m}_{q} \leq \mu \leq \bar{m}_{Q}$ (green bands) for $n_{Q}=n_{\ell}+1=5$ (lower band) and $n_{Q}=n_{\ell}+1=4$ (upper band). The black dashed lines show the prediction for $\mu=\bar{m}_{Q}$ which gives the simple approximation formula in Eq. (15). Right: The $O\left(\alpha_{s}^{3}\right)$ virtual quark mass correction $\delta_{Q, 3}^{(q)}\left(r_{q Q}\right)$ for $n_{Q}=n_{\ell}+1=5$ (red curve). The green band is the prediction for $\delta_{Q, 3}^{(q)}\left(r_{q Q}\right)$ using the method used in the left plot showing excellent agreement to the exact result within errors.

become equivalent due to heavy quark symmetry. The strong cancellation in the sum of both types of corrections in $\delta m_{q}^{(Q \rightarrow q)}\left(\bar{m}_{q}\right)\left(\sim 75 \%\right.$ at $O\left(\alpha_{s}^{2}\right)$ and $\gtrsim 90 \%$ at $O\left(\alpha_{s}^{3}\right)$ for the cases displayed in Fig. 2$)$ thus confirms that the known $O\left(\alpha_{s}^{2}\right)$ and $O\left(\alpha_{s}^{3}\right)$ self-energy corrections coming from virtual quark masses are already dominated by their large order asymptotic behavior.

The series for $\delta m_{b, c}^{(t \rightarrow b)}\left(\bar{m}_{b}, \bar{m}_{c}\right)$ and $\delta m_{c}^{(b \rightarrow c)}\left(\bar{m}_{c}\right)$ converge very well and their $O\left(\alpha_{s}^{3}\right)$ corrections amount to only about $1 \mathrm{MeV}$, thus we can expect that the two types of corrections that enter $\delta m_{b, c}^{(t \rightarrow b)}\left(\bar{m}_{b}, \bar{m}_{c}\right)$ agree even better than $1 \mathrm{MeV}$ at $O\left(\alpha_{s}^{4}\right)$ and beyond. This allows to make an approximate prediction for the yet uncalculated $O\left(\alpha_{s}^{4}\right)$ finite mass corrections from virtual loops of a quark $q$ in the pole- $\overline{\mathrm{MS}}$ mass relations of a heavy quark $Q$ by setting the $O\left(\alpha_{s}^{4}\right)$ correction in $\delta m_{q}^{(Q \rightarrow q)}\left(\bar{m}_{q}\right)$ to zero. The residual $\mu$-dependence vanishes in the formal limit that the virtual quark $q$ mass corrections are entirely dominated by their large order asymptotic behavior and can be used as an uncertainty estimate of our approximation. For $\mu=\bar{m}_{Q}$, we can provide the very simple closed analytic expression

$$
\delta_{Q, 4}^{(q)}\left(r_{q Q}\right) \approx r_{q Q}\left[\delta_{q, 4}^{(q)}(1)-\left(6 \beta_{0}^{\left(n_{Q}\right)} \delta_{q, 3}^{(q)}(1)+4 \beta_{1}^{\left(n_{Q}\right)} \delta_{2}(1)\right) \ln \left(r_{q Q}\right)+12 \delta_{2}(1)\left(\beta_{0}^{\left(n_{Q}\right)} \ln \left(r_{q Q}\right)\right)^{2}\right]
$$

The coefficients $\delta_{q, n}^{(q)}(1)$ describe the corrections from virtual massive loops of the heavy quark $q$ to the $q$ self-energy and are known up to $O\left(\alpha_{s}^{4}\right)$ from the full $a_{n}$ coefficients computed in Refs. [1-8].

In the left plot of Fig. 3 we show the prediction of $\delta_{Q, 4}^{(q)}\left(r_{q Q}\right)$ for the top ( $n_{Q}=n_{t}=5$, lower band) and bottom $\left(n_{Q}=n_{b}=4\right.$, upper band) with a scale variation of $\bar{m}_{b} \leq \mu \leq \bar{m}_{t}$ and $\bar{m}_{c} \leq \mu \leq \bar{m}_{b}$ respectively. The appropriate curves for Eq. (15) are shown as the black dashed lines. The uncertainty amounts to $\pm 3 \%$ (for $r_{q Q} \lesssim 0.1$ ) or smaller (for $r_{q Q}>0.1$ ). The reliability of the prediction and uncertainty estimate was additionally tested by "predicting" the already exactly known $\delta_{Q, 3}^{(q)}\left(r_{q Q}\right)$ using the same method, see the right plot of Fig. 3. The prediction is fully compatible with the exact result and the uncertainty amounts to $\pm 10 \%$ (for $r_{q Q} \lesssim 0.1$ ) or smaller (for $r_{q Q}>0.1$ ).

To conclude it should be mentioned that the prediction of Eq. (15) can be generalized to even higher orders in $\alpha_{s}$ by using the known asymptotic behavior of the coefficients $a_{n}$, as well as to the case of having a larger number of lighter massive quarks. 


\subsection{Pole Mass Differences}

Another interesting application of the RG-setup introduced here is the determination of the difference of the pole masses of two massive quarks. Due to heavy quark symmetry, the differences of two heavy quark pole masses are free of $O\left(\Lambda_{\mathrm{QCD}}\right)$ renormalon ambiguities and can therefore be determined to high precision. The matching corrections discussed above and the R-evolution of the MSR mass allow us to systematically sum logarithms of the mass ratios that would remain unsummed in a fixed-order calculation, and to achieve more precise perturbative predictions [18]. The resulting relations between the top, bottom and charm quark pole masses read

$$
\begin{aligned}
m_{t}^{\text {pole }}-m_{b}^{\text {pole }}= & {\left[\bar{m}_{t}-\bar{m}_{b}\right]+\Delta m_{t}^{(6 \rightarrow 5)}\left(\bar{m}_{t}\right)+\Delta m^{(5)}\left(\bar{m}_{t}, \bar{m}_{b}\right)+\delta m_{b, c}^{(t \rightarrow b)}\left(\bar{m}_{b}, \bar{m}_{c}\right) } \\
m_{b}^{\text {pole }}-m_{c}^{\text {pole }}= & {\left[\bar{m}_{b}-\bar{m}_{c}\right]+\Delta m_{b}^{(5 \rightarrow 4)}\left(\bar{m}_{b}\right)+\Delta m^{(4)}\left(\bar{m}_{b}, \bar{m}_{c}\right)+\delta m_{c}^{(b \rightarrow c)}\left(\bar{m}_{c}\right) } \\
m_{t}^{\text {pole }}-m_{c}^{\text {pole }}= & {\left[\bar{m}_{t}-\bar{m}_{c}\right]+\Delta m_{t}^{(6 \rightarrow 5)}\left(\bar{m}_{t}\right)+\Delta m^{(5)}\left(\bar{m}_{t}, \bar{m}_{b}\right)+\delta m_{b, c}^{(t \rightarrow b)}\left(\bar{m}_{b}, \bar{m}_{c}\right) } \\
& +\Delta m_{b}^{(5 \rightarrow 4)}\left(\bar{m}_{b}\right)+\Delta m^{(4)}\left(\bar{m}_{b}, \bar{m}_{c}\right)+\delta m_{c}^{(b \rightarrow c)}\left(\bar{m}_{c}\right)
\end{aligned}
$$

Each of the mass differences is the sum of universal matching and evolution building blocks which each can be computed to high precision as mentioned at the end of Sec. 3.3. The numerical evaluation using the values given in Ref. [14] for the case $\left(\bar{m}_{t}, \bar{m}_{b}, \bar{m}_{c}\right)=(163,4.2,1.3) \mathrm{GeV}$ gives

$$
\begin{aligned}
& m_{t}^{\text {pole }}-m_{b}^{\text {pole }}=168.169 \pm 0.016 \mathrm{GeV}, \\
& m_{b}^{\text {pole }}-m_{c}^{\text {pole }}=3.331 \pm 0.017 \mathrm{GeV}, \\
& m_{t}^{\text {pole }}-m_{c}^{\text {pole }}=171.500 \pm 0.024 \mathrm{GeV}
\end{aligned}
$$

The uncertainties in the pole mass differences are between 16 and $24 \mathrm{MeV}$ and should be considered as conservative estimates of the theoretical uncertainties due to missing higher order corrections.

\section{Conclusions}

We have provided a renormalization group framework which allows to study the mass effects of virtual massive quark loops in the relation between the pole mass $m_{Q}^{\text {pole }}$ and short-distance masses such as the $\overline{\mathrm{MS}}$ mass $\bar{m}_{Q}(\mu)$ of a heavy quark $Q$, where we mean virtual loop insertions of quarks $q$ with $\Lambda_{\mathrm{QCD}}<m_{q}<m_{Q}$. Special focus was put on the top, where these corrections are most important. In this context it is well-known that the virtual loops of a massive quark act as an infrared cut-off on the virtuality of the gluon exchange that eliminates the effects of that quark from the large order asymptotic behavior of the series. This effect arises from the $O\left(\Lambda_{\mathrm{QCD}}\right)$ renormalon contained in the pole mass which means that the QCD corrections have a linear sensitivity to small momenta that increases with the order in the perturbative expansion.

It was examined (i) how the logarithms of mass ratios that arise in this multi-scale problem can be systematically summed to all orders, (ii) the large order asymptotic behavior and structure of the mass corrections themselves and (iii) the consequences of heavy quark symmetry. E.g. the framework allows to relate the QCD corrections in the top quark pole- $\overline{\mathrm{MS}}$ mass difference $m_{t}^{\text {pole }}-\bar{m}_{t}\left(\bar{m}_{t}\right)$ coming from scales smaller than the bottom mass, to the bottom quark pole- $\overline{\mathrm{MS}}$ mass difference $m_{b}^{\text {pole }}-\bar{m}_{b}\left(\bar{m}_{b}\right)$.

Within the RG framework, we find that the bulk of the lighter virtual quark mass corrections is determined by their large order asymptotic behavior already at $O\left(\alpha_{s}^{3}\right)$, which confirms earlier observations made in Refs. $[19,20]$ and [21]. We used this property to predict the previously unknown $O\left(\alpha_{s}^{4}\right)$ lighter virtual quark mass corrections to within a few percent from the available information on the 
$O\left(\alpha_{s}^{4}\right)$ corrections for massless lighter quarks without an additional loop computation, see Eq. (15). Furthermore we calculated the differences of the top, bottom and charm quark pole masses with a precision of around $20 \mathrm{MeV}$.

\section{Acknowledgments}

We acknowledge partial support by the FWF Austrian Science Fund under the Doctoral Program No. W1252-N27 and the Project No. P28535-N27.

\section{References}

[1] R. Tarrach, Nucl. Phys. B183, 384 (1981)

[2] N. Gray, D.J. Broadhurst, W. Grafe, K. Schilcher, Z. Phys. C48, 673 (1990)

[3] K.G. Chetyrkin, M. Steinhauser, Phys. Rev. Lett. 83, 4001 (1999), hep-ph/9907509

[4] K.G. Chetyrkin, M. Steinhauser, Nucl. Phys. B573, 617 (2000), hep-ph/9911434

[5] K. Melnikov, T.v. Ritbergen, Phys. Lett. B482, 99 (2000), hep-ph/9912391

[6] P. Marquard, L. Mihaila, J.H. Piclum, M. Steinhauser, Nucl. Phys. B773, 1 (2007), hep-ph/0702185

[7] P. Marquard, A.V. Smirnov, V.A. Smirnov, M. Steinhauser, Phys. Rev. Lett. 114, 142002 (2015), 1502.01030

[8] P. Marquard, A.V. Smirnov, V.A. Smirnov, M. Steinhauser, D. Wellmann, Phys. Rev. D94, 074025 (2016), 1606.06754

[9] A.S. Kronfeld, Phys. Rev. D58, 051501 (1998), hep-ph/9805215

[10] I.I.Y. Bigi, M.A. Shifman, N.G. Uraltsev, A.I. Vainshtein, Phys. Rev. D50, 2234 (1994), hep-ph/9402360

[11] M. Beneke, V.M. Braun, Nucl. Phys. B426, 301 (1994), hep-ph/9402364

[12] N. Isgur, M.B. Wise, Phys. Lett. B232, 113 (1989)

[13] M. Beneke, Phys. Rept. 317, 1 (1999), hep-ph/9807443

[14] A.H. Hoang, C. Lepenik, M. Preisser, JHEP 09, 099 (2017), 1706.08526

[15] P. Ball, M. Beneke, V.M. Braun, Nucl. Phys. B452, 563 (1995), hep-ph/9502300

[16] S. Bekavac, A. Grozin, D. Seidel, M. Steinhauser, JHEP 10, 006 (2007), 0708 . 1729

[17] A.H. Hoang, A. Jain, I. Scimemi, I.W. Stewart, Phys. Rev. Lett. 101, 151602 (2008), 0803.4214

[18] A.H. Hoang, A. Jain, C. Lepenik, V. Mateu, M. Preisser, I. Scimemi, I.W. Stewart (2017), 1704.01580

[19] A.H. Hoang, A.V. Manohar, Phys. Lett. B483, 94 (2000), hep-ph/9911461

[20] A.H. Hoang (2000), hep-ph/0008102

[21] C. Ayala, G. Cvetič, A. Pineda, JHEP 09, 045 (2014), 1407. 2128 